\title{
Triple-sleeve lobectomy for non-small cell lung cancer: Lessons from a case
}

Geraud Galvaing, MD, MSc, ${ }^{a}$ Sami Sassi, MD, ${ }^{b}$ Francois Dagenais, MD, ${ }^{\mathrm{c}}$ and Massimo Conti, MD,

Clermont-Ferrand, France, and Québec City, Québec, Canada

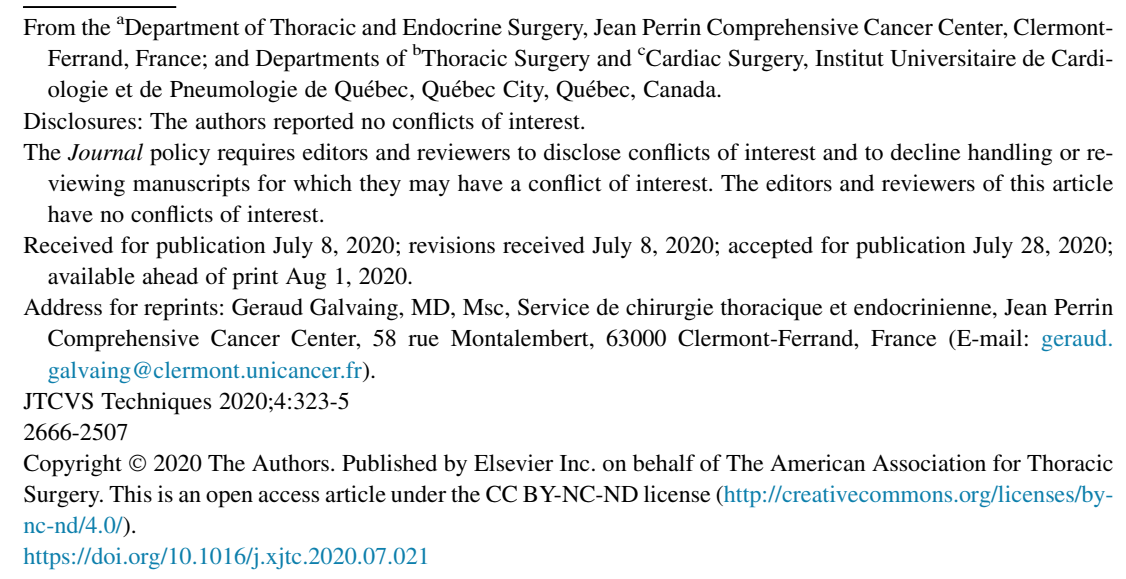

The combination of a bronchial and arterial (double) sleeve lobectomy associated with the prosthetic replacement of the superior vena cava (SVC) has rarely been reported. Herein, we report such a case and highlight operative technique and pitfalls.

\section{CASE DESCRIPTION}

A 66-year-old woman, a heavy smoker, was diagnosed with a large adenocarcinoma originating from the right upper lobe bronchus invading the truncus anterior with close proximity to the SVC (Figure 1). Bronchoscopy confirmed the obstruction of the right upper bronchus by the tumor and a normal mucosa in the intermediate bronchus. Positron emission tomography scan and magnetic resonance imaging of the brain ruled out any nodal invasion or distant metastasis. Lung function tests (forced expiratory volume in 1 second $=87.6 \%$ and diffusing capacity for carbon monoxide $=67.4 \%$ ) indicated the patient could hardly

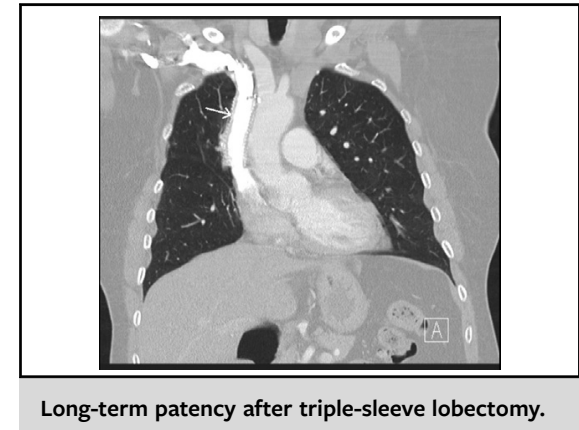

\section{CENTRAL MESSAGE \\ Triple-sleeve lobectomy is technically demanding, but it can offer long-term patency and survival. It should be considered for centrally located tumors in carefully selected patients.}

See Commentaries on pages 326 and 328.

tolerate a pneumonectomy, so she was scheduled for a double-sleeve lobectomy. Our institutional review board waived patient consent regarding this manuscript.

Via a right posterolateral thoracotomy, we first performed a radical hilar and mediastinal lymphadenectomy; frozen section examination turned out to be negative. The pericardium was opened to transect safely the right upper pulmonary vein. The truncus anterior was resected at its origin using a 5/0 nonabsorbable lateral running suture.

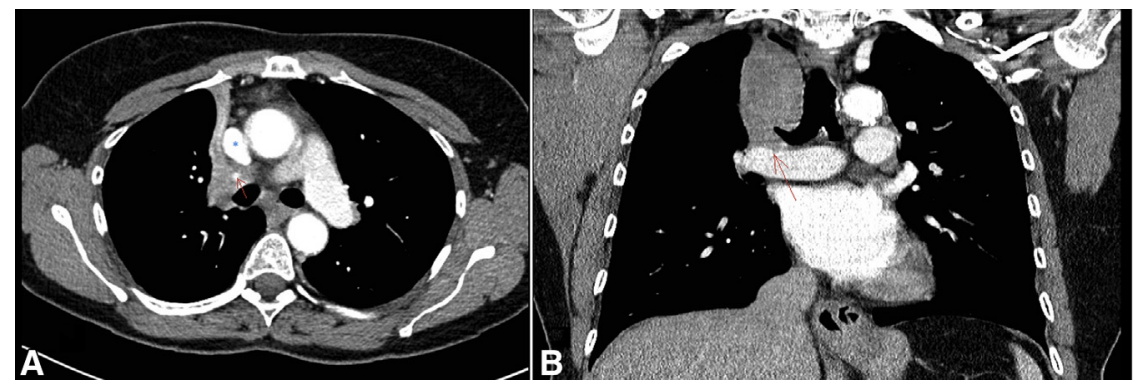

FIGURE 1. A, Preoperative axial computed tomography showing the proximal mass involving the right pulmonary artery (red arrow) and the proximity to the superior vena cava (blue asterisk). B, preoperative coronal computed tomography demonstrating the contact between the mass and the proximal right pulmonary artery (red arrow). 


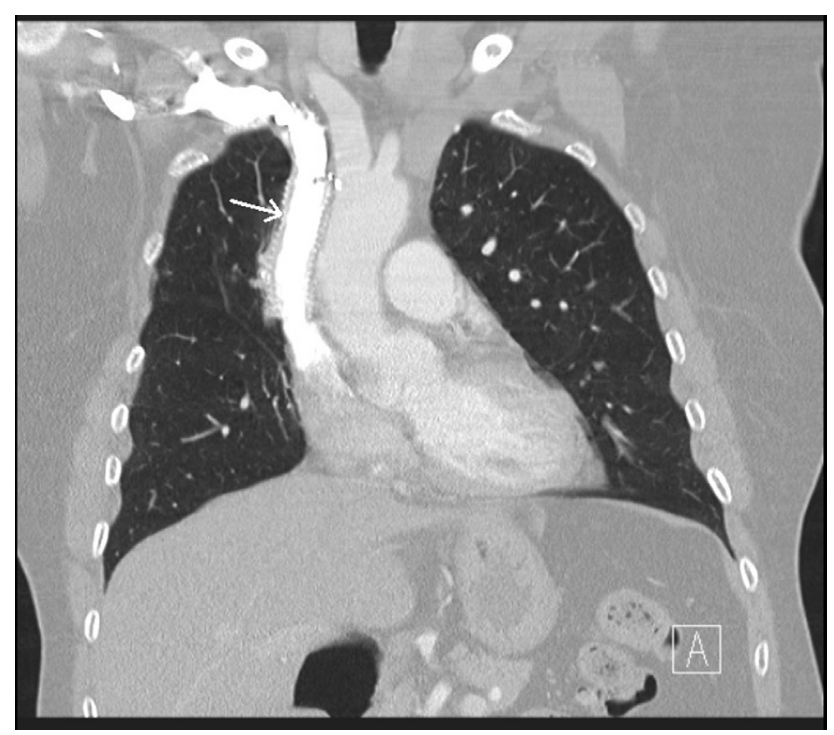

FIGURE 2. Postoperative coronal computed tomography demonstrating superior vena cava graft patency 2 years after the procedure. The ringed polytetrafluoroethylene graft is indicated by the arrow.

Then, the right main bronchus and the bronchus intermedius were transected. Because of the tumor infiltration, a lateral resection of the SVC was performed using a 4/ 0 nonabsorbable running suture. The frozen sections were positive on the bronchial and vascular margins; therefore, an additional section on the right main bronchus was performed. A bovine pericardial patch was added to reconstruct the pulmonary artery and SVC following additional resections. All frozen sections turned out to be negative.

SVC syndrome rapidly appeared. The decision was made, with the cardiac surgeon on site, to replace the SVC. After a 5000-UI heparin infusion, the left innominate vein was ligated and a 14-mm polytetrafluoroethylene vascular graft was sewn between the right innominate vein to the intrapericardial superior vena cava using 2 termino-terminal 5/0 nonabsorbable sutures. The central venous pressure dropped significantly, thus solving the SVC syndrome. The bronchial anastomosis was finally performed and an intercostal muscle flap was interposed between the bronchial and vascular anastomosis.

The patient's postoperative course was marked by a right vocal cord paralysis and a middle lobe pneumonia requiring 3 bronchoscopies and antibiotics. The patient was discharged home on postoperative day 29 with warfarin. The final pathologic report confirmed complete resection of a 4.7-cm pleomorphic carcinoma pT4N2 (4R single station, directly involved by the tumor). Adjuvant chemotherapy was advised, but the patient couldn't tolerate more than 1 infusion. Close follow-up was initiated, and warfarin was switched to aspirin 3 months after the procedure. Successive computed tomography scans confirmed SVC graft

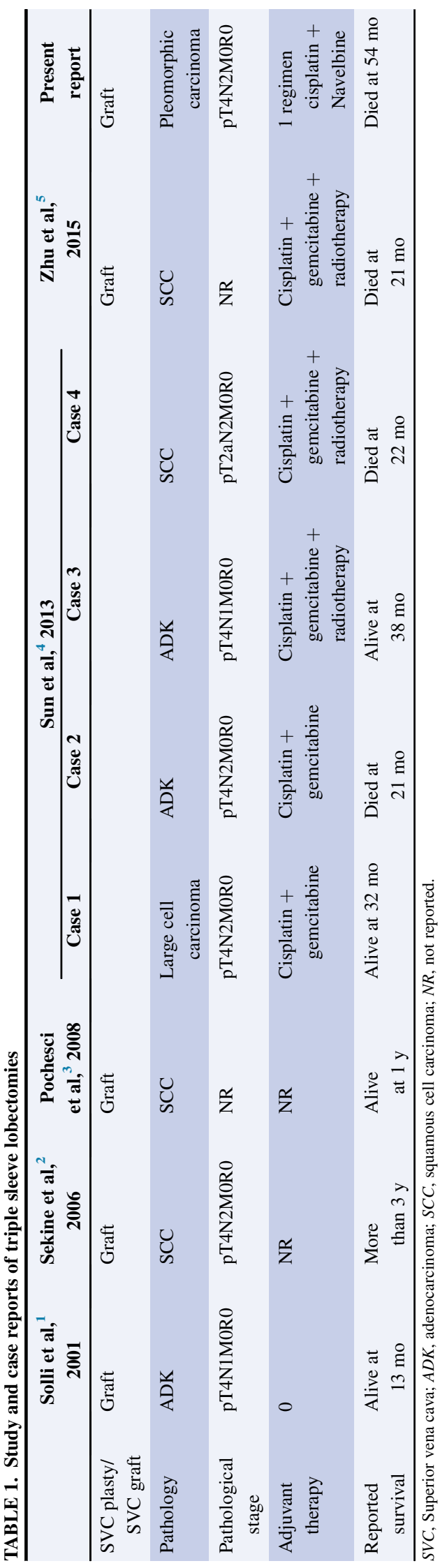


patency (Figure 2). She died 54 months after the surgery for a metastatic cutaneous melanoma.

\section{COMMENT}

Centrally located bronchogenic carcinomas (cT4 tumors) continue to be a challenge for thoracic surgeons, especially when a lung-sparing procedure has to be performed because of the respiratory condition of the patient. Triple reconstruction of the SVC, pulmonary artery, and bronchus, however, has been rarely reported, with only, to the best of our knowledge, 4 case reports ${ }^{1-4}$ and a short series of 4 patients, ${ }^{5}$ as summarized in Table 1 . In the series by Sun and colleagues, ${ }^{5}$ all 4 patients underwent adjuvant treatment, as most were pT4N2, with reported survival up to 3 years. Solli and colleagues ${ }^{1}$ described a triple-sleeve upper bilobectomy classified pT4N1 without adjuvant chemotherapy in a patient who died 13 months after the surgery of distant metastasis. Every reported patient had a complete R0 resection, as they benefited from per-operative frozen section examinations. No prosthetic graft infections were reported despite the nearby bronchial anastomosis. The present case report is to our knowledge the longest survival even with suboptimal adjuvant therapy.

Sewing a prosthetic graft between the right innominate vein and the intrapericardial SVC can be challenging via a right posterolateral thoracotomy; Sekine and colleauges ${ }^{2}$ overpassed this difficulty by performing a median sternotomy after rough closure of the right thoracotomy to get easy access to the superior caval veins. With interdisciplinary collaboration, we were able to perform efficiently and rapidly a difficult SVC graft interposition. This interdisciplinary collaboration is important to emphasize to optimize patient outcomes.

Multi-cT4N0 could be surgical candidates even with limited pulmonary function as long as a complete resection can be achieved. Patient selection is mandatory and interdisciplinary collaboration may be of value to enhance the outcome of such a long and complex procedure.

\section{References}

1. Solli P, Spaggiari L, Grasso F, Pastorino U. Double prosthetic replacement of pulmonary artery and superior vena cava and sleeve lobectomy for lung cancer. Eur J Cardiothorac Surg. 2001;20:1045-8.

2. Sekine Y, Yasufuku K, Motohashi S, Fujisawa T. Triple reconstruction of pulmonary artery, superior vena cava and bronchus for lung cancer. Interact Cardiovasc Thorac Surg. 2006;5:509-10.

3. Poschesci I, Ibrahim M, Vismara LG, Rendina EA. Superior vena cava replacement by the stapled pericardial conduit associated with double sleeve resection of the bronchus and pulmonary artery. Eur J Cardiothorac Surg. 2008;34:673.

4. Zhu D, Qiu X, Zhou Q. Combined double sleeve lobectomy and superior vena cava resection for non-small cell lung cancer with persistent left superior vena cava. Chin J Lung Cancer. 2015;18:718-20.

5. Sun Y, Zheng H, Chen Q, Bao M, Jiang G, Chen C, et al. Triple plasty of bronchus, pulmonary artery, and superior vena cava for non-small cell lung cancer. Ann Thorac Surg. 2013;95:420-4. 